\title{
Image-guided endoscopy: description of technique and potential applications
}

\author{
Marc R. Mayberg, M.D., Eric LaPresto, M.S., and Edwin J. Cunningham, M.D. \\ Seattle Neuroscience Institute, Seattle, Washington; and Department of Neurosurgery, Cleveland \\ Clinic Foundation, Cleveland, Ohio
}

\begin{abstract}
Object. Neuroendoscopic approaches to lesions of the central nervous system and spine are limited by the loss of stereoscopic vision and high-fidelity image quality inherent in the operating microscope. Image-guided endoscopy (IGE) and image-guided surgery (IGS) have the potential to overcome these limitations. The goal of this study was to evaluate IGE for its potential applications in neurosurgery.

Methods. To determine the feasibility of IGE, a rigid endoscope was tracked using an IGS system that provided navigational data for the endoscope tip and trajectory as well as a computer-generated, three-dimensional, virtual representation of the image provided by the endoscope.

The IGE procedure was successfully completed in 14 patients (nine with pituitary adenomas, one with a temporal cavernous malformation, and four with unruptured aneurysms). No complications could be attributed to the procedure. Compared with direct microscopy performed using anatomical landmarks, registration of the endoscope, and virtual image were highly accurate.

Conclusions. This procedure offers many potential advantages for central nervous system and spinal endoscopy. Advances in IGE may enable its application to regions outside the central nervous system as well.
\end{abstract}

KEY WoRDS • endoscopy • image-guided surgery • pituitary adenoma • aneurysm

Image-guided surgery has been widely applied for neurosurgical, ${ }^{1,3,24}$ otolaryngological, ${ }^{11,14}$ and other procedures. ${ }^{8,28}$ This modality provides highly accurate real-time information to the surgeon regarding localization, trajectory, and depth of instrument end, and frequently enables less-invasive approaches to lesions of the brain, skull base, and spine.

Endoscopy has been applied to various neurosurgical procedures, including the resection of intraventricular, ${ }^{25,30}$ sellar and parasellar, ${ }^{15,18}$ intraspinal and paraspinal, ${ }^{4,10}$ extraaxial intracranial, ${ }^{2}$ and vascular lesions. ${ }^{7,23}$ Pituitary tumors and other lesions of the anterior skull base and clivus are particularly well suited to transsphenoidal endoscopic surgery because of the ease of access through the nostril, the working space provided by the sphenoid sinus, and fields of view that are potentially wider and nonlinear compared with those offered by direct microscopy. ${ }^{1}$ Nevertheless, transsphenoidal endoscopy sacrifices the stereoscopic view provided by direct microscopy, and orientation (especially midline) may be more difficult without exposure of anatomical landmarks. For these reasons, many surgeons prefer a transnasal, extramucosal approach in which direct microscopy is used, ${ }^{27}$ because the morbidity related to this approach is comparable to that of endoscopy.

Computer-generated virtual reality representations can be

Abbreviations used in this paper: IGE = image-guided endoscopy; IGS = image-guided surgery; $\mathrm{MR}=$ magnetic resonance; $3 \mathrm{D}=$ three-dimensional. combined with real-world views to produce "augmented reality." ${ }^{2}$ Currently, augmented reality applications in medicine are being developed for neurosurgery, ${ }^{17}$ otolaryngology, ${ }^{11}$ orthopedic surgery, ${ }^{8}$ maxillofacial surgery, and general surgery. ${ }^{6,29}$

To address the relative loss of stereoscopic depth perception and orientation related to endoscopy, we combined IGS with rigid endoscopy to treat various intracranial and sellar lesions. We hypothesized that accurate localization of the endoscope's tip and trajectory would facilitate orientation and enable less-invasive approaches. In addition, coregistration of a computer-rendered 3D virtual representation of the endoscope's field of view would potentially augment depth perception and display healthy and diseased structures that are not directly visible to the endoscope.

\section{CLINICAL MATERIAL AND METHODS}

\section{Endoscopy Equipment}

A 5-mm rigid endoscope (DCI; Storz and Co., Tuttlingen, Germany) was used for all procedures. This endoscope has a digital video output; an automatic rotational orientation feature was disabled to accommodate the coregistration of rendered IGS views. Endoscope angles of $0^{\circ}, 30^{\circ}$, and $70^{\circ}$ were used interchangeably. For lesions of the sella and the parasellar region, a transnasal, transsphenoidal approach was used as previously described. ${ }^{27}$ The osteotomy at the anterior sphenoid bone was enlarged to accommodate 
direct microscopy for verification of anatomical localization. For intracranial lesions (temporal cavernomas and aneurysms), an entry point on the skull was identified using "virtual endoscopy" to visualize the intracranial lesion and choose a trajectory that avoided any intervening vascular structures (see later for more detail). A 3-cm craniotomy was then made at the entry point, and endoscopy was performed as just described during the approach to the lesion.

\section{Image-Guided Surgery}

The CBYON IGS system was used in all cases. Patients underwent placement of between six and eight skin fiducial markers before preoperative MR images were obtained; various MR imaging protocols were used depending on the characteristics of the lesion and the pertinent adjacent anatomy. The patient's head was rigidly fixed and fiducials were registered using standard techniques; registration accuracy was confirmed by identification of skin fiducials and other surface landmarks. The IGS screen was formatted to provide five windows: axial, coronal, and sagittal MR images demonstrating the location and trajectory of the endoscope's tip; endoscope video output; and a 3D rendered image of the virtual endoscopic view. Thresholding techniques were used to highlight the lesion and adjacent healthy structures (arteries and nerves), which were color-coded for representation in the 3D view (Fig. 1). The relative opacity of skin, skull, and brain was adjusted to enable transparency and visualization of highlighted structures.

Virtual endoscopy was performed at the start of the procedure by using the standard IGS pointer, which indicates a rendered 3D view along the trajectory of the pointer-facilitated choice of entry point. The endoscope was fitted with a four-sphere reflective localizer at the camera mounting. The endoscopic image was then coregistered to the virtual image by using a handheld circle grid (Fig. 2A); rotational and in-out movements of the endoscope in the grid confirmed the accuracy of the coregistration (Fig. 2B). Coregistration to the grid was repeated with each change of the endoscope lens.

\section{Patient Population}

Fourteen patients were included in this study, which was conducted between July 2001 and September 2003. Patients were selected according to the suitability of the lesion for endoscopy and IGS. Nine pituitary tumors, one right temporal cavernous malformation, three pericallosal artery

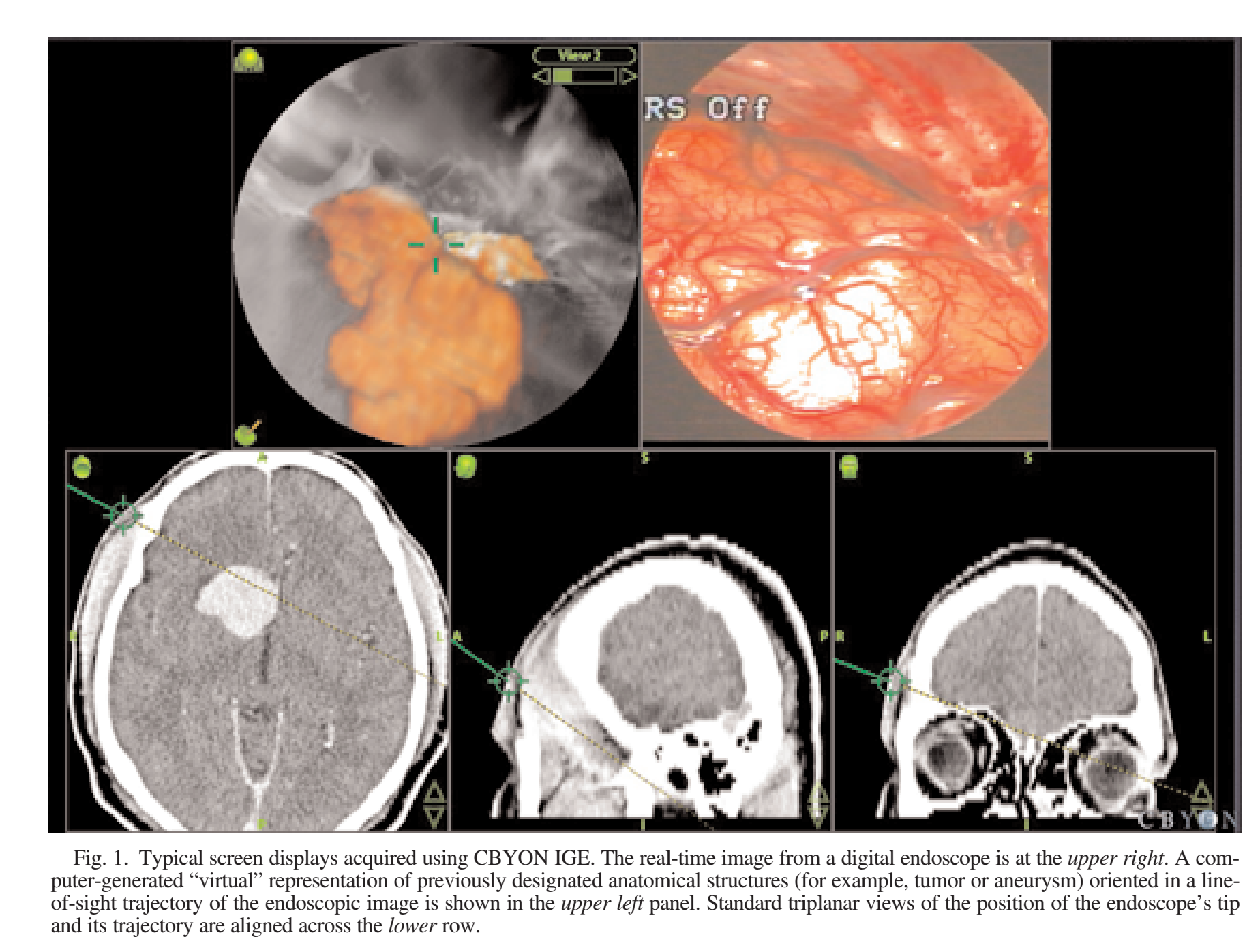




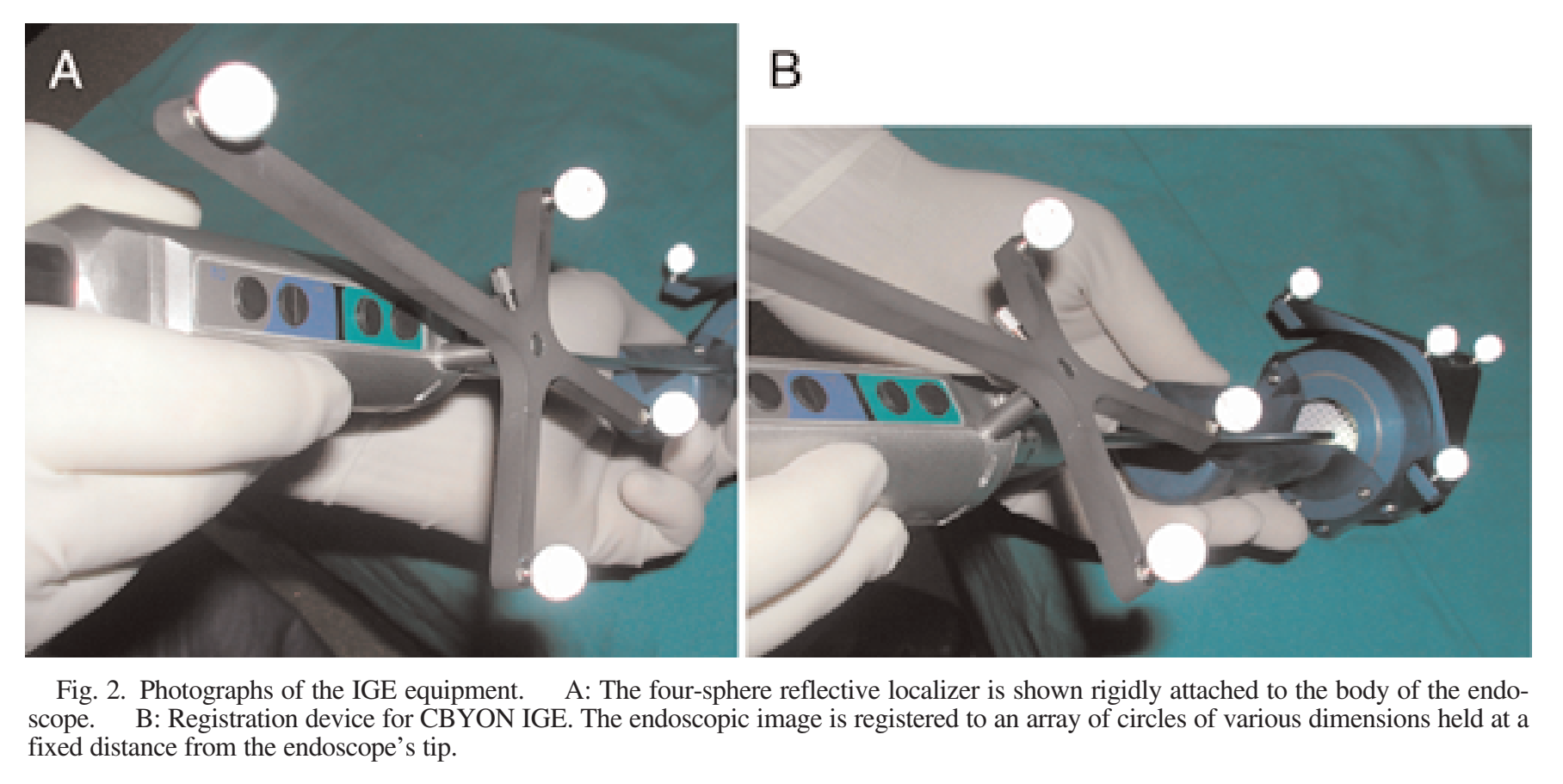

aneurysms, and one middle cerebral artery aneurysm were treated.

\section{Confirmation of IGE Accuracy}

In all cases, once the lesion was identified using IGE, an operating microscope was used to confirm the accuracy of surface landmarks identified with IGE. For the pericallosal aneurysms, microscopy was combined with endoscopy during the aneurysm clip occlusion procedure.

\section{RESULTS}

No complications occurred that were attributable to IGE or otherwise. The initial registration of the IGS system and coregistration of the endoscope added approximately 15 minutes to the length of the operation; an additional 15 to 30 minutes was spent before surgery for image transfer and processing. Complete (gross-total) resections were obtained for seven of nine pituitary tumors. Complete resection of the temporal cavernous malformation and clip occlusion of the aneurysms were confirmed on postoperative MR imaging and intraoperative angiography, respectively.

Excellent accuracy was obtained for IGE views. The position of the endoscope's tip corresponded to surface landmark anatomy with a level of accuracy comparable to standard IGS pointing devices. Similarly, there was an excellent concordance between direct endoscopic views and rendered virtual images, as assessed by surface landmark topography and lesion margins; the accuracy of representation for nonvisualized structures (carotid artery, optic nerves) could not be assessed. Although the time required for final endoscope positioning was not compared with non-IGE approaches, it was the surgeon's impression that IGE significantly reduced the amount of time needed for endoscope placement, facilitated immediate and accurate positioning of the endoscope, and reduced the amount of time required for location and dissection of the cavernoma and aneurysms.

\section{DISCUSSION}

For several reasons, the potential for central nervous system endoscopy has not been fully realized. First, endoscopy for abdominal, thoracic, joint, and cranial sinus procedures is performed through distinct cavities that provide a working space with generally resilient margins that is accessed via relatively noninvasive approaches. In general, current neuroendoscopic approaches have been developed for natural cavities (ventricle, paranasal sinuses, disc space, thoracic cavity), and are applicable to a limited subset of lesions within or immediately adjacent to those cavities. Second, neurosurgeons are uniformly comfortable and adept at microsurgery using the operating microscope, which affords high resolution, excellent illumination, and a stereoscopic view, often through exposures that are not significantly more invasive than those used with endoscopy. Third, neuroendoscopic instrumentation has not been developed to accommodate specific tissue-handling issues (for example, brain retraction) or neurosurgical tasks (for example, aneurysm clip placement, tumor removal, and so on). Nevertheless, the central nervous system and spine may be highly suited to endoscopy, and current limitations should be surmountable through technology development and physician training. ${ }^{24}$

In contrast to endoscopy, neuronavigation using IGS has substantially preceded similar applications in other specialties. In the relatively short interval since its initial descriptions, ${ }^{26}$ IGS has become an integral part of neurosurgical technique. In theory, IGS enables less-invasive and safer approaches to the brain and spine, although these advantages have not been definitively demonstrated. Initial reports on image-guided neuroendoscopy used frame-based 
stereotaxy. ${ }^{5,12,16,30}$ Subsequently, Rhoten, et al., ${ }^{25}$ described the use of a frameless and armless stereotactic system combined with neuroendoscopy. In their study, image guidance facilitated trajectory planning for transventricular procedures and allowed immediate localization and orientation of the endoscope tip. One drawback to this system was the need for intermittent insertion of the stereotactic wand into the endoscope and then replacement of surgical instruments to localize the current endoscope position. Kawamata, et al. ${ }^{19}$ and Konen, et al., ${ }^{20}$ recently overcame this problem by developing an augmented reality navigation system specifically designed for neuroendoscopic procedures. In combination with an optical tracking system, two sets of built-in, infrared light-emitting diodes provided continuous measurement of the tip position and the orientation of the endoscope relative to the patient's head. Three-dimensional virtual images of the anatomy and pathological entities were superimposed on real-time endoscopic live images to provide augmented reality. We found the application of augmented reality image guidance to be particularly useful during repeated transsphenoidal operations in which midline landmarks were absent.

A major disadvantage of IGS has been the need to display navigation data on a workstation screen that is outside the operative field, requiring the surgeon to reorient between the two views. This has been addressed by overlaying navigation data on the operating microscope's field of view, including trajectories and target contours. These overlays, however, generally provide only two-dimensional data, and target contours derived from a single perpendicular plane are less helpful in the typical microscope depth of field. Strategies to inject 3D data using binocular overlays are currently in development. ${ }^{9}$

Another promising graphic interface for neuroendoscopy is the head-mounted, heads-up display. ${ }^{13,21}$ Headmounted systems provide either two-dimensional or 3D images. Three-dimensional systems are further classified as immersive, semiimmersive, and nonimmersive. Immersive systems use a large helmet and do not allow for visualization of the surgical field in conjunction with the 3D image. Semiimmersive 3D systems have head-mounted displays that incorporate two liquid crystal displays, providing binocular views of endoscopic or stereotactic images, and are designed to allow the surgeon simultaneous direct vision of the surgical field. Nonimmersive systems are monitor-based. Recently Levy, et al., ${ }^{21}$ described their semiimmersive monocular system, in which a single highresolution liquid crystal display depicting the endoscopic image was built into headgear worn by the surgeon, and viewed with the surgeon's dominant eye. They successfully used the system in 60 patients, and noted that the headsup display improved orientation within the cisterns or ventricular system, and that it enabled the surgeon to concentrate on the surgical field with rapid access to the endoscopic image.

The IGE procedure provides several unique features that may be of potential benefit to the surgeon. First, the standard navigation coordinates of the endoscope tip and trajectory provide $3 \mathrm{D}$ orientation. Second, the navigation data and augmented reality image provide cues for depth perception. Third, the augmented reality image can display both diseased and healthy structures that are hidden from direct view by soft tissue or bone, and maintain a visual ori- entation during intermittent loss of the endoscope's image fidelity (for example, obscuration by blood). Fourth, visualization of the 3D augmented reality target lesion during placement of the endoscope facilitates rapid and accurate transit and positioning of the instrument. Finally, the accommodation of angled endoscopes enables both direct and virtual inspection of structures tangential to the direct line of vision.

On the other hand, there are several limitations inherent in current IGE procedures. Preoperative imaging, image processing, and registration add time and potential cost to the procedure. As in any IGS, inaccurate registration may result in complications related to a false sense of security on the part of the surgeon, and tissue shift during the procedure produces inaccuracy. In our experience, endoscope and virtual reality registrations were accurate and tissue shift was minimal in the lesions chosen for this approach. The IGE procedure did not add significant time to the operation. In fact, IGE appeared to reduce operating time by facilitating rapid and accurate endoscope placement. Despite the wider field of view afforded by angled scopes, the system at present is restricted to rigid endoscopes, and thus to a linear approach for the trajectories. Finally, this study showed feasibility for the technology, but did not demonstrate its safety or efficacy.

Future development of IGE may enable several new applications of neuroendoscopy. For example, $120^{\circ}$ or greater angles on the IGE devices might enable direct and virtual views of the opposite side of lesions (for example, aneurysms, acoustic neuromas, vascular compression of cranial nerves) during microsurgical or endoscopic treatment. Also, flexible IGS-tracked endoscopes may allow endoscopic procedures to be completed via nonlinear approaches, such as through the subarachnoid or epidural spaces. Finally, high-resolution virtual endoscopy may augment diagnostic image interpretation and surgical or endovascular planning. Ultimately, IGE and head-mounted displays may eliminate the operating microscope altogether as well as the need for brain retraction or the wide exposures required to provide linear trajectories to lesions of the brain or spine.

\section{References}

1. Apuzzo ML, Heifetz MD, Weiss MH, et al: Neurosurgical endoscopy using the side-viewing telescope. J Neurosurg 46: 398-400, 1977

2. Barnett GH: Surgical management of convexity and falcine meningiomas using interactive image-guided surgery systems. Neurosurg Clin N Am 7:279-284, 1996

3. Barnett GH, Maciunas RJ, Roberts DW (eds): Computer Assisted Neurosurgery. New York: Marcel Dekker, 2004

4. Bolger C, Wigfield C: Image-guided surgery: applications to the cervical and thoracic spine and a review of the first 120 procedures. J Neurosurg (Spine 2) 92:175-180, 2000

5. Caemaert J, Abdullah J: Diagnostic and therapeutic stereotactic cerebral endoscopy. Acta Neurochir 124:11-13, 1993

6. Decampli WM: Video-assisted thoracic surgical procedures in children. Semin Thorac Cardiovasc Surg Pediatr Card Surg Annu 1:61-74, 1998

7. Dempsey PK, Kondziolka D, Lunsford LD: Stereotactic diagnosis and treatment of pineal region tumors and vascular malformations. Acta Neurochir 116:14-22, 1992

8. DiGioia AM III, Jaramaz B, Colgan BD: Computer assisted orthopaedic surgery: image guided and robotic assistive technologies. Clin Orthop Relat Res 345:8-16, 1998 
9. Edwards PJ, King AP, Maurer CR Jr, de Cunha DA, et al: Design and evaluation of a system for microscope-assisted guided interventions (MAGI). IEEE Trans Med Imaging 19: 1082-1093, 2000

10. Foley KT, Smith MM: Image-guided spine surgery. Neurosurg Clin N Am 7:171-186, 1996

11. Freysinger W, Gunkel AR, Thumfart WF: Image-guided endoscopic ENT surgery. Eur Arch Otorhinolaryngol 254: 343-346, 1997

12. Goodman RR: Magnetic resonance imaging-directed stereotactic endoscopic third ventriculostomy. Neurosurgery 32: 1043-1047, 1993

13. Harrington JA, Harris DM, Katzir A: Progress in biomedical optics. Proc Biomed Optoelectronic Instrumentation 2396: 2-6, 1995

14. Hauser R, Westermann B, Probst R: Noninvasive tracking of patient's head movements during computer-assisted intranasal microscopic surgery. Laryngoscope 107:491-499, 1997

15. Heilman CB, Shucart WA, Rebeiz EE: Endoscopic sphenoidotomy approach to the sella. Neurosurgery 41:602-607, 1997

16. Hellwig D, Bauer BL: Endoscopic procedures in stereotactic neurosurgery. Acta Neurochir Suppl 52:30-32, 1991

17. Iseki H, Masutani Y, Iwahara M, et al: Volumegraph (overlaid three-dimensional image-guided navigation). Clinical application of augmented reality in neurosurgery. Stereotact Funct Neurosurg 68:18-24, 1997

18. Jho HD, Alfieri A: Endoscopic endonasal pituitary surgery: evolution of surgical technique and equipment in 150 operations. Minim Invasive Neurosurg 44:1-12, 2001

19. Kawamata T, Iseki H, Shibasaki T, et al: Endoscopic augmented reality navigation system for endonasal transsphenoidal surgery to treat pituitary tumors: technical note. Neurosurgery 50: 1393-1397, 2002

20. Konen W, Scholz M, Tombrock S: The VN project: endoscopic image processing for neurosurgery. Comput Aided Surg 3: 144-148, 1998

21. Levy ML, Day JD, Albuquerque F, et al: Heads-up intraoperative endoscopic imaging: a prospective evaluation of techniques and limitations. Neurosurgery 40:526-530, 1997
22. Maurer CR Jr, Sauer F, Hu B, et al: Augmented reality visualization of brain structures with stereo and kinetic depth cues: system description and initial evaluation with head phantom, in Mun SK (ed): Proceedings of SPIE: Medical Imaging 2001: Visualization, Display, and Image-Guided Procedures, Volume 4319. Bellingham, WA: SPIE, 2001, pp 445-456

23. Origitano TC, Anderson DE: CT angiographic-guided frameless stereotactic-assisted clipping of a distal posterior inferior cerebellar artery aneurysm: technical case report. Surg Neurol 46:450-454, 1996

24. Perneczky A, Fries G: Endoscope-assisted brain surgery: part 1evolution, basic concept, and current technique. Neurosurgery 42:219-225, 1998

25. Rhoten RLP, Luciano MG, Barnett GH: Computer-assisted endoscopy for neurosurgical procedures: technical note. Neurosurgery 40:632-637, 1997

26. Roberts DW, Strohbehn JW, Hatch JF, et al: A frameless stereotaxic integration of computerized tomographic imaging and the operating microscope. J Neurosurg 65:545-549, 1986

27. Roux FX, Page P, Nataf F, et al: Intérêt de la voie d'abord endonasale pour l'exérèse des adénomed hypophysaires Ann Endocrinol 63:187-192, 2002

28. Spirn PW, Shah RM, Steiner RM, et al: Image-guided localization for video-assisted thoracic surgery. J Thorac Imaging 12: 285-292, 1997

29. Wagner A, Millesi W, Watzinger F, et al: Clinical experience with interactive teleconsultation and teleassistance in craniomaxillofacial surgical procedures. J Oral Maxillofac Surg 57: 1413-1418, 1999

30. Zamorano L, Chavantes C, Dujovny M, et al: Stereotactic endoscopic interventions in cystic and intraventricular brain lesions. Acta Neurochir Suppl 54:69-76, 1992

Manuscript received May 19, 2005.

Accepted in final form June 16, 2005.

Address reprint requests to: Marc Mayberg, M.D., Seattle Neuroscience Institute, 1600 Jefferson Avenue, Suite 620 Seattle, Washington 98104. email: marc.mayberg@swedish.org 